\title{
Characterization of social cognition impairment in multiple sclerosis
}

\author{
M. Neuhaus ${ }^{a}$, S. Bagutti ${ }^{a}$, Ö. Yaldizlib, D. Zwahlenc ${ }^{\text {, S. Schaub }}$, B. Frey ${ }^{d}$, B. Fischer-Barnicol ${ }^{\text {b }}$, \\ J.-M. Burgunder ${ }^{d}$ (D), M.-D. Martory ${ }^{e}$, J. Pöttgen ${ }^{\dagger}$ J.-M. Annoni ${ }^{\mathrm{a}, *}$ and I.-K. Pennerg,*
}

\begin{abstract}
${ }^{\mathrm{a}}$ Neurology Unit, University of Fribourg, Fribourg; ${ }^{\mathrm{b}}$ Department of Neurology, University Hospital Basel, Basel; ${ }^{\mathrm{c}}$ Department of Cognitive Psychology, University of Basel, Basel; ${ }^{\mathrm{d}}$ Department of Neurology, University Hospital Berne, Berne; ${ }^{\mathrm{e}}$ Neuropsychology Unit, University Hospital of Geneva, Geneva, Switzerland; ${ }^{\mathrm{f}}$ Institut für Neuroimmunologie und Multiple Sklerose, Klinik und Poliklinik für Neurologie, Universitätsklinikum Hamburg-Eppendorf, Deutschland, Hamburg; and ${ }^{\mathrm{g}}$ Cogito Center for Applied Neurocognition and Neuropsychological Research and Department of Neurology, University Hospital Düsseldorf, Düsseldorf, Germany
\end{abstract}

\author{
Keywords: \\ affect recognition, \\ fatigue, multiple \\ sclerosis, social \\ cognition, theory of \\ mind
}

\begin{abstract}
Background and purpose: Multiple sclerosis (MS) has been associated with deficits in social cognition. However, little is known about which domains of social cognition are predominantly affected and what other factors are associated with it. The aim was (i) to characterize social cognition deficit in a group of MS outpatients and (ii) to relate impairment in social cognition to overall cognitive status, depression and fatigue.

Methods: Thirty-five MS patients (mean disease duration 12.9 years, median Expanded Disability Status Scale (EDSS) 3 and 34 healthy controls (HCs) were examined using the German version of the Geneva Social Cognition Scale to measure different domains of social cognition. Standard neuropsychological testing was applied to all patients and to 20 HCs. Patient-reported outcomes included questionnaires for fatigue, depression, anxiety and executivebehavioural disturbances.

Results: The mean social cognition raw score was lower in the MS patients compared to the HCs $(86.5 \pm 8.7$ vs. $91.2 \pm 5.9, P=0.005 ; d=0.6)$ and did not correlate with EDSS or disease duration. The difference was driven by facial affect recognition and the understanding of complex social situations (14\% and $23 \%$ of patients respectively under the cut-off). The impairment in these two tasks did not correlate with general cognitive performance or depression but with fatigue.

Conclusions: The impairment in our group was restricted to high order and affective social cognition tasks and independent of general cognitive performance, EDSS, disease duration and depression. Fatigue correlated with social cognition performance, which might be due to common underlying neuronal networks.
\end{abstract}

\section{Introduction}

Social cognition (SC) concerns the processing of information that influences our behaviour towards other people. It relies on the knowledge of unwritten social rules and the ability to infer from given information, guess what others might be feeling and correctly judge a situation [1]. It is sub-served by a large brain

Correspondence: J.-M. Annoni, University of Fribourg, CH-1708

Fribourg, Switzerland (tel.: +41 2642681 30; fax: + 412642681 35; e-mail: jean-marie.annoni@unifr.ch).

*Equal contribution. network including the temporo-parietal, medial prefrontal and anterior cingulate cortex [1]. Theory of mind (ToM) is one factor of SC, referring to the ability to make inferences about the mental status of other people and to understand their emotions and beliefs [1].

Social cognition abilities can be divided into various subdomains, such as facial affect recognition, understanding of someone else's state of mind/ToM (false belief tasks) or comprehension of complex social situations (faux pas tasks). These subdomains can be grouped into categories, such as 'verbal/non-verbal' 
depending on their verbal demands and 'emotional/ cognitive' depending on the complexity of their emotional components [2]. The level of complexity varies across tasks. 'Social cognition stories', 'faux pas' and facial affect recognition are considered high order ToM tasks [3], whereas 'false belief' tasks are considered less complex, appearing earlier in normal development.

Functional cognitive and affective alterations associated with impaired frontal-subcortical connectivity play an important role in the everyday disabilities of multiple sclerosis (MS) patients [4]. Given the large network implicated in SC, and as other conditions with disruption of subcortical-frontal connectivity have been associated with SC deficits [5], MS is expected to have an impact on this process [6]. Most studies (19/23, Appendix S3, Table S1) showed that $\mathrm{SC}$ is impaired in MS [7-14]. However, it remains unclear which subdomains are predominantly affected, since many of these studies $[10,14]$ included only one aspect $(13 / 23)$ or did not directly compare the different domains of SC (3/23) [11].

Little is known about factors influencing SC performance in MS patients. Depression is more frequent in MS patients than in the general population and fatigue is one of the most common symptoms of MS. Both have been thought to interfere with cognitive testing [15]. Whilst literature on the effect of fatigue on SC is rare, an effect of major depression on SC abilities has been described [16]. As SC differs meaningfully from other aspects of cognitive functionality
[17], people with MS might have difficulties in interpersonal contexts even in the absence of marked executive dysfunctions and physical disability.

The aim of the present study was to characterize SC deficits in MS patients and to assess whether or not impairment in certain subdomains is associated with disease duration, physical or overall cognitive deficits, depression and fatigue.

\section{Participants and methods}

The study was approved by the Ethics Committee of North West Switzerland (Reference 49/12). Written informed consent by subjects/next-of-kin was provided by all patients and controls.

\section{Patients}

Thirty-nine MS patients were recruited from the MS outpatient clinic of the University Hospital, Basel, and neurological practices in the northwest of Switzerland.

Inclusion criteria were a diagnosis of MS or clinically isolated syndrome according to the revised McDonald criteria 2005 [18]; Expanded Disability Status Scale (EDSS) [19] score $\leq 7.0$; free of corticosteroid treatment and relapses for at least 2 months; fluency in German. Exclusion criteria were severe cognitive impairment including the inability to participate in cognitive testing; major depression; medication or comorbidities that may affect cognition (e.g. schizophrenia or bipolar disorder leading to hospitalization).



Figure 1 Social cognition score in patients and controls. The dotted line indicates the cut-off. The "o" and "*" indicate outliers. 
Four patients withdrew their consent due to scheduling problems or because of the long duration $(2 \mathrm{~h})$ of the testing sessions. In total, 35 patients were tested between November 2012 and January 2013 either in the Institute for Psychology, Basel, or at the University Hospital Basel and are included in this analysis. Subject characteristics are given in Table 1 .

Thirty-four age-, gender- and education-matched healthy individuals from Basel and Berne served as controls (HCs). HCs had no known neurological diseases and did not take any drugs that affect cognitive function. Each rater was instructed by a senior expert neurologist or neuropsychologist (JMA, IKP) in a training session.

\section{Geneva Social Cognition Scale (GeSoCS)}

An original German version of the GeSoCS [20], used for clinical assessment of SC in neurological disorders, was used. Information on the translation process and the German version of the GeSoCS is given in the Supporting material (Appendices S1, S2 and S4) (http://www.unifr.ch/neurology/fr/clinic/pubclnic).

The test consisted of the following subtests: (i) 'social cognition stories', verbal stories, including a faux pas task; (ii) a short version of Baron Cohen's 'reading the mind in the eyes' (MIE) test, a facial affect recognition task; (iii) 'cartoon stories', non-verbal false belief tasks; (iv) 'inference test' short stories where conclusions have to be drawn from given information; (v) 'absurd stories', short situations without social aspect; (vi) 'temporal rules', an executive functions task.

A maximum of 100 points could be achieved. The test duration was $30 \mathrm{~min}$. Details are given elsewhere [20].

\section{Standard neuropsychological test battery}

A 90 min neuropsychological test was applied to MS patients and to $20 \mathrm{HCs}$. It included the Verbal Learning and Memory Test [21], the Brief Visuospatial Memory Test revised [22], semantic and phonemic verbal fluency [23], Digit Span and Corsi-Block from the Wechsler Memory Scale [24] and the Symbol Digit Modalities Test [25].

\section{Questionnaires}

Fatigue and depression/anxiety were assessed by the Fatigue Scale for Motor and Cognitive Functions (FSMC) [26] and the Hospital Anxiety and Depression Scale [27]. To measure behavioural changes close relatives were asked to complete the Dysexecutive Questionnaire [28].

\section{Statistical analysis}

Data were presented as mean \pm standard deviation (SD) or median (range) depending on the distribution. The chi-squared test was used to compare categorical variables. The Mann-Whitney $U$ test or the $t$ test for independent samples was used to compare outcome measures between groups and the Spearman $\rho$ or Pearson correlation tests to assess associations between variables depending on the normality of the data (Shapiro-Wilk test). Results were further analysed using a linear regression model with demographic data (age, gender, education) as independent covariates. Because of the non-normal distribution of data, significant results were confirmed by bootstrap analysis (case resampling, $n=1000$ ). Cut-off values were defined as the mean value minus two standard deviations in the

Table 1 Demographic data of MS patients and controls

\begin{tabular}{|c|c|c|c|}
\hline & $\begin{array}{l}\text { Healthy } \\
\text { controls }\end{array}$ & MS patients & Significance $(P)$ \\
\hline$N$ & 34 & 35 & \\
\hline Age in years, mean (SD) & $43.9(12.5)$ & $43.8(12.13)$ & 0.91 \\
\hline Female & $22 / 34(64.7 \%)$ & $22 / 35(60 \%)$ & 0.69 \\
\hline \multicolumn{4}{|l|}{ Level of education } \\
\hline Obligatory school education only (11 years) & 0 & 1 & 0.49 \\
\hline Apprenticeship & 20 & 19 & \\
\hline $\begin{array}{l}\text { Thirteen years school education and } \\
\text { university admission qualification }\end{array}$ & 2 & 5 & \\
\hline Completed university study & 12 & 10 & \\
\hline EDSS median (range) & NA & $3.0(0-6.5)$ & \\
\hline Disease duration in years, mean (SD) & NA & $12.9(9.6)$ & \\
\hline Clinical course of MS $(n)$ & NA & $\begin{array}{l}\text { Relapsing-remitting } 25 \text {; primary progressive } 8 \text {; } \\
\text { secondary progressive } 2\end{array}$ & \\
\hline
\end{tabular}

MS, multiple sclerosis; EDSS, Expanded Disability Status Scale. 
control group. Given the exploratory nature of this study and the potential problems associated with formally correcting for multiple comparisons [29], flagged results using the conventional significance threshold $(P<0.05)$ are presented. SPSS version 21 for statistical analysis (Chicago, IL, USA) was used.

\section{Results}

\section{Social cognition scores in patients versus controls}

Patients showed lower total SC scores than HCs (86.5 \pm 8.7 vs. $91.2 \pm 5.9, P=0.005$, post hoc calculated statistical power $0.81, d=0.63$; fig. 1 ). The difference remained significant after adjusting for age, gender and education using a linear regression model (adjusted $P=0.008)$. Three patients $(8.6 \%)$ had lower scores than the cut-off value $(<79.3)$. No difference was found between relapsing and progressive MS $(P=0.55)$.

The differences between patients and HCs were driven by the subtests 'social cognition stories' $(P=0.006, d=0.64)$ (Table 2$)$ and $\operatorname{MIE}(P=0.035$, $d=0.57)$, again remaining significant after adjusting for age, gender and education using a linear regression model $(P=0.012$ and 0.004 , respectively). The number of patients who scored below the cut-off in 'social cognition stories' and MIE was eight $(23 \%)$ and five $(14 \%)$. Sixteen $(46 \%)$ patients scored below the cutoff in at least one subtest.

\section{Association between neurological disability, cognitive functions and social cognition scores}

There was no correlation between the total SC score and EDSS or disease duration. The neuropsychological test scores in the patient group were marginally lower than in HCs without reaching statistical significance (Table 3). The difference between MS patients

Table 2 Median and range for results in the total score and subtests of the GeSoCS (all non-normally distributed)

\begin{tabular}{llll}
\hline & $\begin{array}{l}\text { HCs } \\
(n=34)\end{array}$ & $\begin{array}{l}\text { MS } \\
(n=35)\end{array}$ & $\begin{array}{l}\text { Significance } \\
(P)\end{array}$ \\
\hline $\begin{array}{l}\text { Social cognition } \\
\text { total score } \\
\text { Social cognition } \\
\quad \text { stories }\end{array}$ & $20(16-20)$ & $20(14-20)$ & $0.006^{*}$ \\
$\quad \begin{array}{l}\text { Reading the mind } \\
\text { in the eyes }\end{array}$ & $16(10-20)$ & $14(4-18)$ & $0.035^{*}$ \\
$\begin{array}{l}\text { Cartoon stories } \\
\text { Inference test }\end{array}$ & $20(17-20)$ & $19.5(12-20)$ & 0.148 \\
$\begin{array}{l}\text { Absurd stories } \\
\text { Temporal rules }\end{array}$ & $10(14-20)$ & $18(10-20)$ & 0.604 \\
& $10(6-10)$ & $10(6-10)$ & 0.275 \\
\end{tabular}

*Significant results $(P>0.05)$. GeSoCS, Geneva Social Cognition Scale; HC, healthy control; MS, multiple sclerosis. and controls remained significant excluding patients who scored below the cut-off in two or more of the neuropsychological tests $(n=30$ remaining, total score $P=0.012$ ).

In the patients' group, 'social cognition stories' and MIE did not correlate with any other neuropsychological test scores. The global SC score correlated with verbal memory $(\rho=0.384, P=0.025)$, visuospatial memory $(\rho=0.472, \quad P=0.01)$ and verbal fluency $(\rho=0.381, P=0.024)$.

\section{Associations between social cognition, fatigue and depression}

Fatigue and depression scores were higher in patients than controls (Table 4).

There was no correlation between SC and depression scores in MS patients. The difference in SC between MS patients and HCs remained significant even when depression was considered (corrected $P=0.027$ ).

Twenty-two patients suffered from fatigue according to the validated cut-off values [26]; 14 were classified as severe.

Total and cognitive fatigue were correlated with SC ( $\rho=-0.424, P=0.016$ ), confirmed by linear regression analysis $(P=0.001)$ and bootstrap. When corrected for fatigue, the differences between MS patients and controls in the SC score were no longer significant (total score $P=0.36$, 'social cognition stories' $P=0.2$, MIE $P=0.19$ )

\section{Discussion}

Consistent with most published studies (Appendix S3, Table S1) SC impairment was found in MS patients compared with HCs on a group level.

The prevalence of this impairment in our cohort, including patients with a mild-to-moderate disability

Table 3 Mean values (SD) for results in neuropsychological tests

\begin{tabular}{|c|c|c|c|}
\hline Neuropsychological test & $\begin{array}{l}\text { MS } \\
(n=35)\end{array}$ & $\begin{array}{l}\text { Controls } \\
(n=20)\end{array}$ & Significance $(P)$ \\
\hline $\begin{array}{l}\text { Symbol Digit } \\
\text { Modalities Test }\end{array}$ & $50.2(16.5)$ & $57.4(13.1)$ & 0.85 \\
\hline $\begin{array}{l}\text { Verbal Learning and } \\
\text { Memory Test }(n=34)\end{array}$ & $58.7(11.3)$ & $60.1(6.06)$ & 0.57 \\
\hline $\begin{array}{l}\text { Brief Visuospatial } \\
\text { Memory Test }\end{array}$ & $25.3(6.7)$ & $25.1(6.9)$ & 0.91 \\
\hline $\begin{array}{l}\text { Phonological } \\
\text { fluency: words }\end{array}$ & $21.2(7.8)$ & $23.7(6.7)$ & 0.23 \\
\hline $\begin{array}{l}\text { Semantic } \\
\text { fluency: animals }\end{array}$ & $32.3(9.4)$ & $35.7(9.2)$ & 0.19 \\
\hline Digit Span & $14.0(3.5)$ & $15.8(3.6)$ & 0.08 \\
\hline Block Span & $16.3(3.2)$ & $17.4(2.9)$ & 0.19 \\
\hline
\end{tabular}


Table 4 Median (range) and significance of between-group differences for results in questionnaires

\begin{tabular}{lrrl}
\hline & & & \multicolumn{1}{l}{ Significance } \\
& MS & \multicolumn{1}{l}{ Controls } & $(P)$ \\
\hline Fatigue total score & $52(21-94)$ & $32(20-62)$ & $<0.001^{*}$ \\
$\quad$ Motor fatigue & $32.5(11-47)$ & $17(10-29)$ & $0.005^{*}$ \\
Cognitive fatigue & $23.5(10-48)$ & $12.5(10-33)$ & $0.000^{*}$ \\
Anxiety & $5(0-14)$ & $4(0-9)$ & 0.058 \\
Depression & $3(0-12)$ & $1(0-5)$ & $0.000^{*}$ \\
Executive-behavioural & $9.5(0-55)$ & $10.5(0-27)$ & 0.846 \\
disturbances & & & \\
\hline
\end{tabular}

* Significant results $(P>0.05)$

(median EDSS 3), was 9\%, reaching 24\% for facial affect recognition. A prevalence of up to $66 \%$ [9] has been described for impairment in facial affect recognition. This variability may be due to influences by different neurological deficits or patterns of lesion load and suggests that SC abilities should be tested in each patient.

The strength of the present study is that different dimensions of SC were investigated. Until now, mostly one-dimensional tests have been used. A study by Jehna and colleagues [30], for example, tested only one aspect of SC and found no difference between MS patients and controls. This underlines the importance of using a tool like the GeSoCS, which provides a broader view of performance in different categories.

Two subtests most sensitive to differentiate patients from HCs were found: 'social cognition stories' and MIE. These findings are supported by a recent review [31] on ToM in MS, which also found deficits in overall ToM performance as well as facial affect recognition and the interpretation of social situations. The performance of MS patients in faux pas tasks, however, was conserved but with great heterogeneity in the data on this point.

Both facial affect recognition and faux pas tasks are considered complex ToM tasks [2]. Our results suggest that physicians should carefully choose the tests for MS patients. In our population the tests most sensible to detect changes in SC performance are high order tests.

Our data support evidence on impairment in MS patients in both verbal and non-verbal ToM $[7,10]$. The most affected tasks in our study were the verbal 'social cognition stories' and the non-verbal MIE. Both tasks focus on the affective component of ToM, whereas for example false belief tasks rely more on cognitive aspects of ToM [2]. A deficit in emotional recognition and alexithymia in MS patients has been discussed [10,14] and can be the underlying cause for a disruption of affective ToM functions.

Social cognition deficit can occur independently or secondary to deficits in other cognitive domains [31].
The driving subtests were found to be independent, suggesting that SC, although not entirely independent of other cognitive functions, represents a separate entity of socio-cognitive functioning. The reported correlations $[8,10]$ between SC and other cognitive domains may correspond to a mediating role between executive dysfunction and ToM deficits.

Statistically, the difference in SC between patients and controls was not independent of fatigue. However, this does not necessarily mean that fatigue is confounding our results; fatigue and SC may share similar pathophysiological mechanisms. Both fatigue and SC impairment can be caused by a widespread brain network dysfunction and are associated with reduced function of the right prefrontal and anterior cingulate cortex and structural loss of fibre integrity in the frontal white matter [1,32]. In previous studies, $\mathrm{SC}$ in MS patients was independent from fatigue $[9,13]$. However, a direct comparison with our results is difficult, as these studies used different fatigue scales (Modified Fatigue Impact Scale versus FSMC) and neither of them stated the prevalence of fatigue in their MS group. Future studies should further include validated and detailed scales of fatigue to better understand its impact on SC.

\section{Limitations}

The number of subjects chosen for our study was sufficient to depict the difference in SC between the patient and control groups. It was too small, however, for any further secondary analysis. The relatively low number of patients and controls also increased the risk for type 2 errors. The mild level of overall cognitive disability in this group may have influenced the results, especially the lack of association between general cognitive impairment and SC performance. Larger studies including patients with more severe cognitive impairment are warranted.

The GeSoCS has not been validated in German. However, the ability to differentiate patients from controls in most of its subtests is well established [20]. The scale is too long for quick screening in MS patients and should be used as part of a clinical neuropsychological testing or be adapted to the diseases, choosing for example the most sensitive of its subtests.

\section{Conclusions}

Impairment in our group was restricted to high order and affective SC tasks, such as affect recognition and faux pas. This finding might be useful when considering new simple evaluation tools for daily practice. 
Although not entirely independent of other cognitive functions, SC deficit appears to be a separate entity of socio-cognitive impairment. The impairment occurs independently of neurological disability and disease duration; therefore physicians and nurses need to be aware of it at any stage of the disease. Fatigue correlated with SC performance, which might reflect intricate underlying neuronal networks.

\section{Acknowledgements}

This work was supported by a Swiss National Science Foundation Grant No. 325130_156937 to Jean-Marie Annoni. The authors would like to thank Professor Ludwig Kappos for his expertise and Lea Jost for helping with the statistics.

\section{Disclosure of conflicts of interest}

The authors declare no financial or other conflicts of interest.

\section{Supporting Information}

Additional Supporting Information may be found in the online version of this article:

Appendix S1 Description of the translation process. Appendix S2 Instructions to the GeSoCS for testers. Appendix S3 Review of the studies on social cognition in MS.

Table S1 Review of the studies on social cognition in MS.

Appendix S4 Evaluation sheet for the GeSoCS.

\section{References}

1. Frith CD, Frith U. Social cognition in humans. Curr Biol 2007; 17: R724-R732.

2. Muller F, Simion A, Reviriego E, et al. Exploring theory of mind after severe traumatic brain injury. Cortex 2010; 46: 1088-1099.

3. Stone VE, Gerrans P. What's domain-specific about theory of mind? Soc Neurosci 2006; 1: 309-319.

4. Simioni S, Schluep M, Bault N, et al. Multiple sclerosis decreases explicit counterfactual processing and risk taking in decision making. PLoS One 2012; 7: e50718.

5. Kana RK, Libero LE, Moore MS. Disrupted cortical connectivity theory as an explanatory model for autism spectrum disorders. Phys Life Rev 2011; 8: 410-437.

6. Mike A, Strammer E, Aradi M, et al. Disconnection mechanism and regional cortical atrophy contribute to impaired processing of facial expressions and theory of mind in multiple sclerosis: a structural MRI study. PLoS One 2013; 8: e82422.
7. Banati M, Sandor J, Mike A, et al. Social cognition and theory of mind in patients with relapsing-remitting multiple sclerosis. Eur J Neurol 2010; 17: 426-433.

8. Ouellet J, Scherzer PB, Rouleau I, et al. Assessment of social cognition in patients with multiple sclerosis. $J$ Int Neuropsychol Soc 2010; 16: 287-296.

9. Henry A, Tourbah A, Chaunu M-P, et al. Social cognition impairments in relapsing-remitting multiple sclerosis. J Int Neuropsychol Soc JINS 2011; 17: 11221131.

10. Henry JD, Phillips LH, Beatty WW, et al. Evidence for deficits in facial affect recognition and theory of mind in multiple sclerosis. J Int Neuropsychol Soc 2009; 15: $277-$ 285.

11. Kraemer M, Herold M, Uekermann J, et al. Theory of mind and empathy in patients at an early stage of relapsing-remitting multiple sclerosis. Clin Neurol Neurosurg 2013; 115: 1016-1022.

12. Pöttgen J, Dziobek I, Reh S, et al. Impaired social cognition in multiple sclerosis. J Neurol Neurosurg Psychiatry 2013; 84: 523-528.

13. Roca M, Manes F, Gleichgerrcht E, et al. Cognitive but not affective theory of mind deficits in mild relapsing-remitting multiple sclerosis. Cogn Behav Neurol 2014; 27: 25-30.

14. Prochnow D, Donell J, Schäfer R, et al. Alexithymia and impaired facial affect recognition in multiple sclerosis. J Neurol 2011; 258: 1683-1688.

15. Chiaravalloti ND, DeLuca J. Cognitive impairment in multiple sclerosis. Lancet Neurol 2008; 7: 1139-1151.

16. Weightman MJ, Air TM, Baune BT. A review of the role of social cognition in major depressive disorder. Front Psychiatry 2014; 5: 1-13.

17. Stone VE, Hynes CA. Real-World Consequences of Social Deficits: Executive Functions, Social Competencies, and Theory of Mind in Patients with Ventral Frontal Damage and Traumatic Brain Injury. New York: Oxford University press, 2011.

18. Polman CH, Reingold SC, Banwell B, et al. Diagnostic criteria for multiple sclerosis: 2010 revisions to the McDonald criteria. Ann Neurol 2011; 69: 292-302.

19. Kurtzke JF. Rating neurologic impairment in multiple sclerosis: an Expanded Disability Status Scale (EDSS). Neurology 1983; 33: 1444-1452.

20. Martory M-D, Pegna AJ, Sheybani L, et al. Assessment of social cognition and theory of mind: initial validation of the Geneva Social Cognition Scale. Eur Neurol 2015; 74: 288-295.

21. Helmstaedter C, Lendt M, Lux S. Verbaler Lern- und Merkfähigkeitstest. Göttingen, Germany: Beltz Test GmbH, 2001.

22. Benedict RHB, Schretlen D, Groninger L, Dobraski M, Shpritz B. Revision of the Brief Visuospatial Memory Test: studies of normal performance, reliability, and validity. Psychol Assess 1996; 8: 145-153.

23. Aschenbrenner A, Tucha OLK. RWT Regensburger Wortflüssigkeits-Test. Handanweisung. Göttingen: Hogrefe Verlag, 2000.

24. Härting $\mathrm{C}$, Markowitsch $\mathrm{HJ}$, Neufeld $\mathrm{H}$, Calabrese $\mathrm{P}$, Diesinger K, Kessler J. Wechsler Gedächtnis Test - Revidierte Fassung (WMS-R). Berne: Huber, 2000.

25. Smith A. Symbol Digit Modalities Test. Los Angeles: Western Psychological Services, 1995. 
26. Penner I, Raselli C, Stöcklin M, et al. The Fatigue Scale for Motor and Cognitive Functions (FSMC): validation of a new instrument to assess multiple sclerosis-related fatigue. Mult Scler 2009; 15: 1509-1517.

27. Hermann-Lingen C, Buss U, Snaith R. Hospital Anxiety and Depression Scale - Deutsche Version (HADS-D). Berne: Huber, 1995.

28. Simblett SK, Bateman A. Dimensions of the Dysexecutive Questionnaire (DEX) examined using Rasch analysis. Neuropsychol Rehabil 2011; 21: 1-25.

29. Perneger TV. What's wrong with Bonferroni adjustments. BMJ 1998; 316: 1236-1238.
30. Jehna M, Neuper C, Petrovic K, et al. An exploratory study on emotion recognition in patients with a clinically isolated syndrome and multiple sclerosis. $\mathrm{Clin} \mathrm{Neu}$ rol Neurosurg 2010; 112: 482-484.

31. Cotter J, Firth J, Enzinger C, et al. Social cognition in multiple sclerosis. A systematic review and meta-analysis. Neurology 2016; 87: 1727-1736.

32. Roelcke U, Kappos L, Lechner-Scott J, et al. Reduced glucose metabolism in the frontal cortex and basal ganglia of multiple sclerosis patients with fatigue: a $18 \mathrm{~F}$ fluorodeoxyglucose positron emission tomography study. Neurology 1997; 48: 1566-1571. 